\title{
Tomato Susceptibility to Root-Knot Nematodes Requires an Intact Jasmonic Acid Signaling Pathway
}

\author{
Kishor K. Bhattarai, ${ }^{1}$ Qi-Guang Xie, ${ }^{1}$ Sophie Mantelin, ${ }^{1}$ Usha Bishnoi, ${ }^{1}$ Thomas Girke, ${ }^{2}$ \\ Duroy A. Navarre, ${ }^{3}$ and Isgouhi Kaloshian ${ }^{1}$ \\ ${ }^{1}$ Department of Nematology and ${ }^{2}$ Department of Botany and Plant Sciences, Center for Plant Cell Biology, University of \\ California, Riverside 92521, U.S.A.; ${ }^{3}$ United States Department of Agriculture-Agricultural Research Service, Washington \\ State University, Prosser 99350, U.S.A.
}

Submitted 5 February 2008. Accepted 29 April 2008.

\begin{abstract}
Responses of resistant (Mi-1/Mi-1) and susceptible (mi-1/ mi-1) tomato (Solanum lycopersicum) to root-knot nematodes (RKNs; Meloidogyne spp.) infection were monitored using cDNA microarrays, and the roles of salicylic acid (SA) and jasmonic acid (JA) defense signaling were evaluated in these interactions. Array analysis was used to compare transcript profiles in incompatible and compatible interactions of tomato roots $24 \mathrm{~h}$ after RKN infestation. The jail and defl tomato mutant, altered in JA signaling, and tomato transgenic line $N a h G$, altered in SA signaling, in the presence or absence of the RKN resistance gene $\mathrm{Mi}$ 1 , were evaluated. The array analysis identified 1,497 and 750 genes differentially regulated in the incompatible and compatible interactions, respectively. Of the differentially regulated genes, $37 \%$ were specific to the incompatible interactions. $N a h G$ affected neither $M i-1$ resistance nor basal defenses to RKNs. However, jail reduced tomato susceptibility to RKNs while not affecting $\mathrm{Mi}-1$ resistance. In contrast, the $\operatorname{def} 1$ mutant did not affect RKN susceptibility. These results indicate that JA-dependent signaling does not play a role in $\mathrm{Mi}$-1-mediated defense; however, an intact JA signaling pathway is required for tomato susceptibility to RKNs. In addition, low levels of SA might be sufficient for basal and $M i-1$ resistance to RKNs.
\end{abstract}

Additional keywords: Coi-1, plant defense, proteinase inhibitors.

Root-knot nematodes (RKNs; Meloidogyne spp.) are sedentary endoparasitic nematodes with a wide host range and are considered one of the most damaging nematode pests to crops worldwide (Koenning et al. 1999). The second-stage juveniles (J2) are the infective stage that penetrates the root tips and migrates to the vasculature, where they start intimate interactions with the plant. RKNs induce an elaborate change in the cell architecture in susceptible hosts. Cells undergo repeated karyogenesis without cytogenesis in response to secretions of the esophageal glands. These feeding sites are known as giant cells and serve as a nutrient source for the nematode and nutrient sink for the plant. Cells around the giant cells undergo concurrent hyperplasia and hypertrophy, which are manifested as galls on plant roots. Female nematodes become saccate, repro-

Corresponding author: Isgouhi Kaloshian; Telephone: +1.951.827.3913; Fax: +1.951.827.4434; E-mail: isgouhi.kaloshian@ucr.edu

* The $e$-Xtra logo stands for "electronic extra" and indicates that two supplemental tables are available online. duce parthenogenetically, and lay eggs on the root surface in a protective gelatinous matrix (Williamson and Gleason 2003).

The tomato gene $M i-1$ confers resistance to three species of RKN, Meloidogyne arenaria, M. incognita, and $M$. javanica (Dropkin 1969). $M i-1$ also confers resistance to potato aphids, Macrosiphum euphorbiae (Rossi et al. 1998), and two biotypes of whiteflies, Bemisia tabaci and B. tabaci biotype B (Nombela et al. 2003). Mi-1-mediated defense responses to nematodes are associated with induction of the hypersensitive response (HR) (Dropkin 1969), one of the hallmarks of gene-for-gene resistance. $\mathrm{Mi}-\mathrm{I}$ belongs to the largest class of resistance genes cloned to date and encodes a protein with a coiled-coil domain, nucleotide-binding, and leucine-rich repeat motifs (Milligan et al. 1998). In addition to $M i-1$, nematode and insect resistance in tomato depends on the presence of another gene, Rme1, the identity of which is still unknown and appears to be specific for $\mathrm{Mi}-1$ function (Martinez de Ilarduya et al. 2001, 2004). Full $M i-1$ function to aphids and nematodes also requires the heat shock protein $H s p 90-1$ and the co-chaperonelike gene Sgt1 (Bhattarai et al. 2007a). Moreover, Mi-1-mediated aphid resistance requires members of mitogen-activated protein kinase (MAPK) cascades, including the MAPK kinase LeMKK2 and MAPKs LeMPK2, LeMPK1, and LeMPK3 (Li et al. 2006). The role of these genes in RKN resistance is currently unknown.

Evidence indicates that, in a plant, a vast array of genes work coordinately to defend themselves from the invading pathogen. During incompatible interactions, the plant cells undergo rapid reprogramming and many physiological changes such as ion fluxes, oxidative burst, and the rapid and transient production of reactive oxygen species intermediates are observed (Glazebrook 2005; Hammond-Kosack and Parker 2003). Cell wall reinforcement around the infection site and production of the antibiotic compounds are detected, which are often accompanied by HR. Three biochemical compounds, salicylic acid (SA), jasmonic acid (JA), and ethylene (ET), are known to regulate both basal and resistance $(R)$ gene-mediated defense responses (Glazebrook 2005; Kunkel and Brooks 2002). In general, JA and ET signaling pathways seem to work synergistically whereas SA and JA/ET signaling pathways are antagonistic and communicate mainly by negative cross talk, indicating a trade off between these pathways. However, cooperation and sequential positive interactions have also been reported between SA and JA/ET pathways (Bostock 2005; Rojo et al. 2003).

A role for the SA signaling pathway in $\mathrm{Mi}$-1-mediated resistance to RKNs and aphids has been identified. Partial loss of Mi-1-mediated RKN resistance was observed in genetically re- 
sistant tomato roots expressing NahG (Branch et al. 2004). These experiments were performed using transgenic hairy roots transformed with Agrobacterium rhizogenes and nematode assays were performed in monoaxenic tissue cultures. Similarly, loss of potato aphid resistance was observed in transgenic plants expressing $N a h G$ in the $M i-l$ background ( $\mathrm{Li}$ et al. 2006). In both cases, application of the SA analog Benzothiadiazole rescued the susceptible phenotype to RKNs and aphids (Branch et al. 2004; Li et al. 2006). In addition, rapid transcripts accumulation of Pathogenesis related $1(P R-1)$, an SA-inducible defense marker, was associated with the incompatible tomato-aphid interactions compared with the compatible interactions (Martinez de Ilarduya et al. 2003). Proteinase inhibitors (Pin) Pin1 and Pin2 transcripts, regulated by the JA signaling, also accumulated after potato aphids feeding in both compatible and incompatible interactions (Martinez de Ilarduya et al. 2003). However, in spite of activation of the JA responsive genes, no role for JA signaling was identified in the $M i-1$ mediated aphid resistance (Bhattarai et al. 2007b).

During the past decade, considerable progress has been made in understanding the complex changes of plant gene expression elicited by RKN infection during compatible interactions (Gheysen and Fenoll 2002). Genes involved in cell cycle, plant development, phytohormone synthesis, and nodule formation have been implicated in this interaction (Bird and Koltai 2000; Gheysen and Fenoll 2002). Contrary to the compatible interaction, limited information exists about the defense responses in the incompatible interactions to RKNs (Williamson and Kumar 2006). Initial attempts to identify genes differentially regulated in the $\mathrm{Mi}-1$-mediated resistance to RKNs were not successful (Lambert et al. 1999). Using differential screening of a tomato root cDNA library, genes regulated in both compatible and incompatible responses to $\mathrm{RKN}$ infection were isolated, suggesting considerable overlap between basal plant defense responses and $\mathrm{Mi}$ - 1 -mediated resistance.

To identify genes differentially regulated in the incompatible $M i-1-\mathrm{RKN}$ interactions, we performed microarray analysis. In this process, we also identified genes differentially regulated during the compatible tomato-RKN interactions. Our results indicated that all three SA-, JA-, and ET-regulated defense pathways are activated in both compatible and incompatible interactions; however, their level of activation was higher during incompatible interaction. We used tomato mutants and $N a h G$ transgene to assess functionally the role of the JA and

\section{Up-regulated Down-regulated}



Fig. 1. More genes are differentially regulated during incompatible than compatible tomato-RKN interactions. Venn diagrams of tomato genes differentially expressed during compatible ('Moneymaker,' $\mathrm{mi} / \mathrm{mi}$ ) and incompatible ('Motelle,' Mi-1/Mi-1) tomato-RKN interactions. For each genotype, pooled RNA from four biological replicates for a total of two replicates for each treatment was used. RNAs from mock-infected roots were compared with RNAs from root knot nematode-infected roots. Statistical significance of $P<0.05$ was considered. The number of genes upregulated by at least 2 -fold and downregulated by at least 1.5 -fold are presented.
SA pathways in RKN defense. Our results indicated a role for JA-dependent signaling in susceptibility to RKNs but not in Mi-1-mediated defense.

\section{RESULTS}

More genes are differentially regulated in the incompatible than

in the compatible tomato-RKN interactions.

To determine the early transcript profile during compatible and incompatible tomato-RKN interactions and to identify genes that are differentially regulated, we used a TOM1 cDNA microarray containing 12,860 tomato expressed sequence tag (EST) clones. To obtain tomato root tissue highly enriched for RKN infection, a previously developed semi-in vitro tomato infection system was used. Although this inoculation system delivered the $\mathrm{J} 2$ behind the root tips, nematodes need time to find and penetrate the root tips and migrate to the vascular cylinder where they initiate feeding. After feeding initiation, plant defense responses are triggered and HR is seen. In this system, initial symptoms of HR were observed $24 \mathrm{~h}$ after nematode infection; therefore, this time point was selected for this study (data not shown). Four biological replicate experiments were performed. RNAs from two biological replicates were pooled before cDNA synthesis. Using linear model for microarray data analysis (Limma) (Smyth 2005), we identified differentially expressed genes between RKN-infected root samples and mock-infected roots based on $P$ value $(P<0.05)$. Adjusted $P$ value from the Limma was used as the primary criteria while fold change was used as secondary criteria to identify the differentially expressed genes. A twofold change cut off was used for differentially upregulated clones, whereas 1.5 -fold was used for the downregulated ones. We considered 1.5-fold for downregulation because, at more stringent criteria (for example, at twofold), we obtained very low numbers of clones downregulated in both compatible $(n=62)$ and incompatible $(n=$ 81 ) interactions. Low numbers of greater than 1.5 -fold downregulated genes are also reported in other plant-pathogen systems (Bonshtien et al. 2005; Navarro et al. 2004; Puthoff et al. 2003).

A number of cDNA clones derived from the same gene were arrayed on the TOM1 chip. In our analysis, we grouped the clones that represented the same gene and we refer to them as

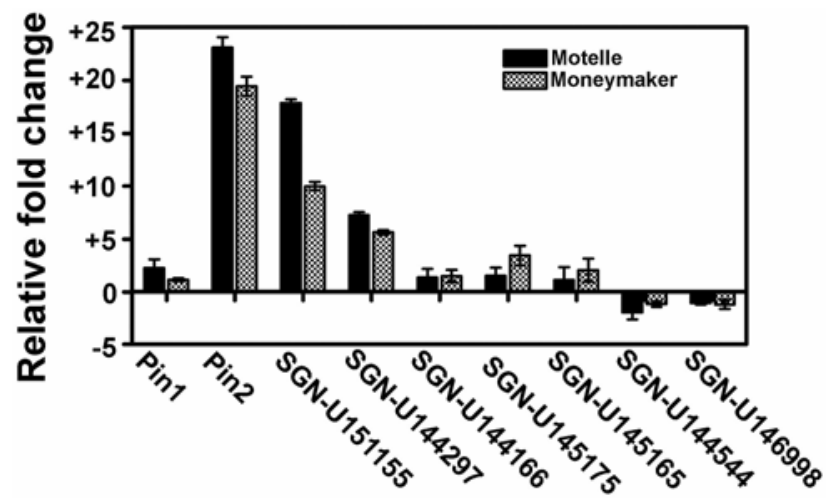

Fig. 2. Quantitative polymerase chain reaction analysis of selected tomato genes. The relative fold change of genes was determined in root-knot nematode-infected compared with mock-infected roots. SGN annotations are: SGN-U151155 = expressed protein, SGN-U144297 = endochitinase precursor, SGN-U144166 = endo- $\beta$ mannanase, SGN-U145175 = cellulose synthase isolog, SGN-U145165 = NTGP4, SGN-U144544 = ribosomal protein L27, and SGN-U146998 = MADS16. For each genotype, two biological replicates were used and three technical replicates per biological replicate were performed. The expression levels of the genes were determined relative to $U b i 3$. 
a single gene. Our analysis indicates that more genes were activated rather than suppressed during both incompatible and compatible tomato-RKN interactions (Fig. 1). In addition, more genes were differentially regulated in the incompatible interactions than the compatible interactions. In all, 1,497 genes in the incompatible interactions and 750 genes in the compatible interactions were differentially expressed based primarily on $P$ value $(P<0.05)$, regardless of fold change. We further ranked these genes based on fold changes. In total, 880 genes were twofold upregulated and 332 were 1.5-fold downregulated during the incompatible interactions, whereas 677 genes were twofold upregulated and 62 were 1.5 -fold downregulated during the compatible interactions (Fig. 1). More than half $(51.2 \%)$ of the differentially regulated genes in the incompatible interactions were unique to this interaction. In the compatible interactions, although genes exclusively differentially regulated were identified $(20.0 \%)$, the great majority of the differentially regulated genes were also differentially regulated in the incompatible interactions (Fig. 1).

To confirm the results obtained from the microarray analysis, 9 differentially expressed genes were selected for reversetranscription real-time quantitative polymerase chain reaction (qPCR). According to microarray analysis, these genes represent five groups: upregulated only in incompatible interactions (SGN-U151155 and SGN-U144297), upregulated in both interactions (SGN-U144166 and SGN-U145175), upregulated only in compatible interactions (SGN-U145165), downregulated only in incompatible interactions (SGN-U144544), and downregulated only in compatible interactions (SGNU146998). We also tested the expression of Pin1 and Pin2. In qPCR, the pattern of these differentially regulated genes was similar to the pattern obtained in the microarray analysis except, in qPCR, slightly higher fold changes were observed for all upregulated clones (Fig. 2).

\section{Functional classification}

of the differentially regulated genes.

To understand the physiological and biological processes that are inherent to the tomato-RKN interactions, genes differentially expressed during both compatible and incompatible interactions were functionally categorized based on Solanaceae Genomics Network and Gene Ontology Consortium annotations (Fig. 3). A large portion (29 and $18 \%$ up- and downregulated, respectively, in compatible, and 35 and $28 \%$ up- and downregulated, respectively, in incompatible) of these genes encoded proteins with insufficient similarity to proteins with known function and, therefore, were categorized as unknowns. Genes with miscellaneous functions represent another large group. These are genes with functions other than the presented functional categories.

During the incompatible interactions, among the known functional groups, the largest group upregulated encoded transcription-related proteins $(9 \%)$, followed by signaling $(6 \%)$ and defense-related (6\%) proteins (Fig. 3). Transcripts of transcription-related $(8 \%)$, signaling $(8 \%)$, and protein biosynthesis-related $(8 \%)$ proteins were the largest three functional

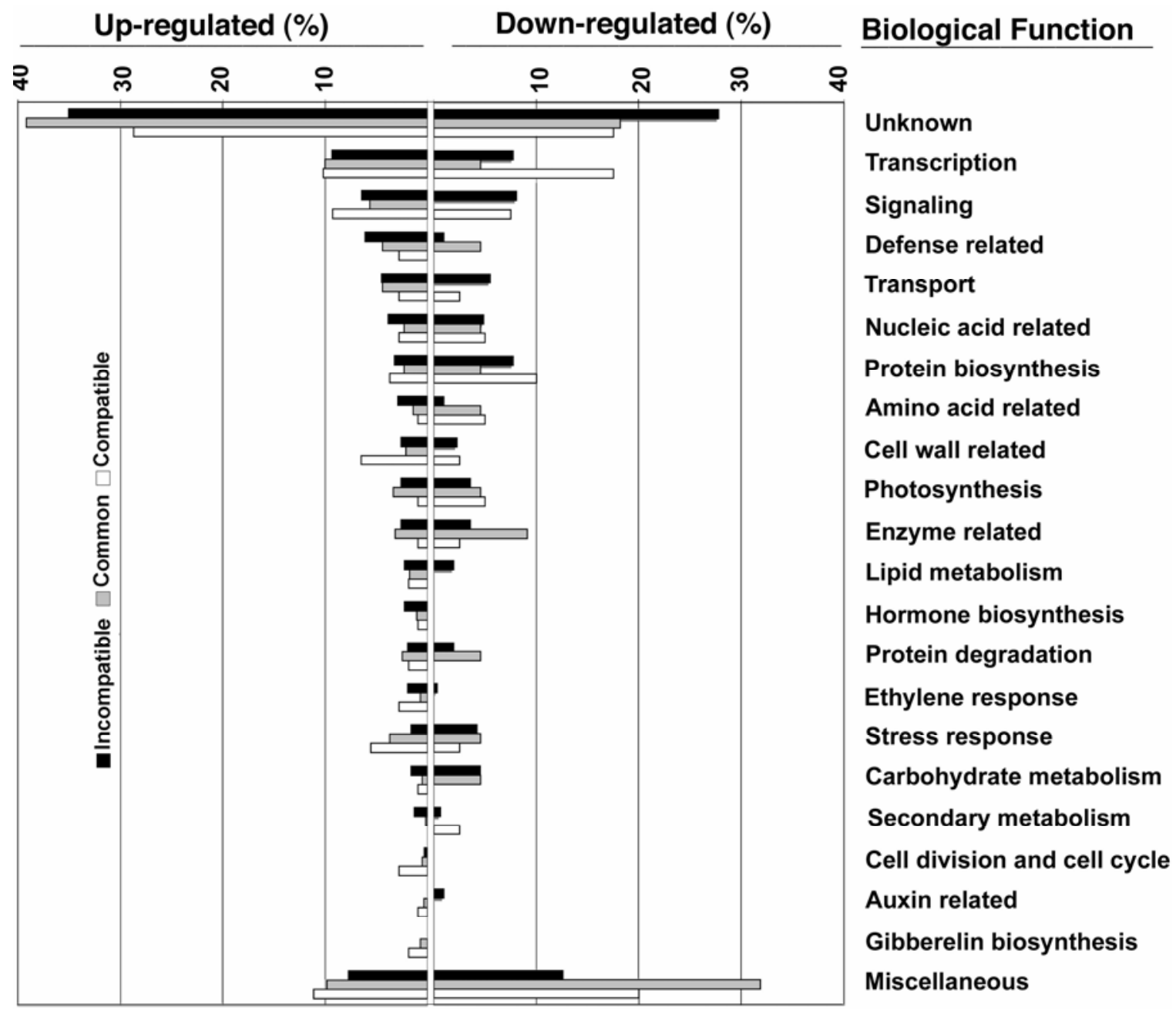

Fig. 3. Genes of diverse functions are regulated in tomato by root-knot nematode (RKN) infections. Functional categories of genes differentially expressed during compatible ('Moneymaker,' $\mathrm{mi} / \mathrm{mi}$ ) and incompatible ('Motelle,' Mi-1/Mi-1) tomato-RKN interactions. 
groups involved in the downregulation. During the compatible interactions, the largest group upregulated encoded transcription-factor-related (10\%), signaling (9\%), and cell-wall-related $(6 \%)$ proteins. Transcription-related $(18 \%)$, protein biosynthesis-related $(10 \%)$, and signaling $(8 \%)$ proteins were the top three functional groups involved in the downregulation during

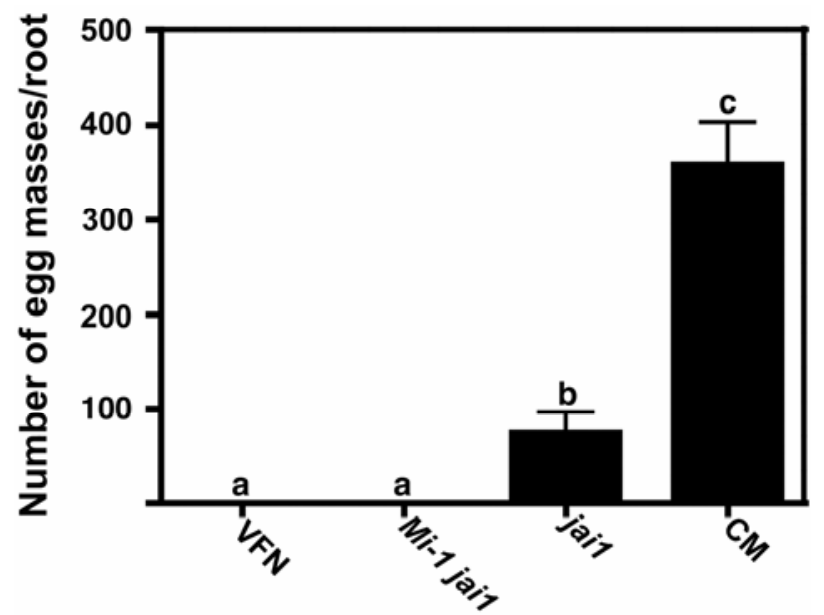

Fig. 4. Nematode reproduction on $\mathrm{Mi}-1$ jail plants and wild-type parents. Five-week-old plants were inoculated with 3,000 root-knot nematode second-stage juveniles and plants were evaluated 8 weeks after inoculation. Error bars indicate standard error of the mean, where bars with different letters denote a significant difference at $P<0.001$. Experiment was performed twice with similar results. Results from one experiment are shown. the compatible interactions (Fig. 3). Transcripts of transcription-related $(10 \%)$, signaling $(6 \%)$, transport $(4 \%)$, stressresponse-related $(4 \%)$, and defense-related $(4 \%)$ proteins were also among the groups commonly activated in both compatible and incompatible interactions (Fig. 3).

\section{JA signaling pathway has a role in basal defense but not} in $\mathrm{Mi}$-1-mediated resistance to RKNs.

The microarray analysis indicated that RNAs of genes involved in JA biosynthesis and genes regulated by JA accumulated in both compatible and incompatible tomato-RKN interactions (Fig. 2), suggesting a role for the JA-regulated signaling pathway in nematode defense. To identify the role of JA in basal and $M i-1$-mediated defenses to RKNs, we used the JA signaling jail mutant, which has a recessive mutation in the Coi-1 gene (Li et al. 2001, 2004). The jail mutant is able to produce endogenous JA but is impaired in JA perception. To address the role of JA in $M i-1$ RKN resistance, $M i-1$ jail plants (Bhattarai et al. 2007b), tomato cv. VFN (Mi-1 Jail), the source of $M i-1$, and the jail wild-type susceptible parent Castlemart (CM; mi Jail) were challenged with RKNs. RKNs were unable to reproduce on $\mathrm{Mi}-1$ jail plants and the numbers of egg masses on these plants were not significantly $(P<$ 0.001 ) different from those on the genetically resistant VFN plants but were significantly different than those on the jail mutant or the wild-type CM (Fig. 4). Although jail and CM plants were both susceptible to RKNs, there was a significantly $(P<0.05)$ lower number of egg masses on jail roots than on the wild-type parent CM (Fig. 4). These findings suggested that JA does not have a role in $\mathrm{Mi}$-1-mediated defense but has a role in susceptibility to RKNs in tomato.
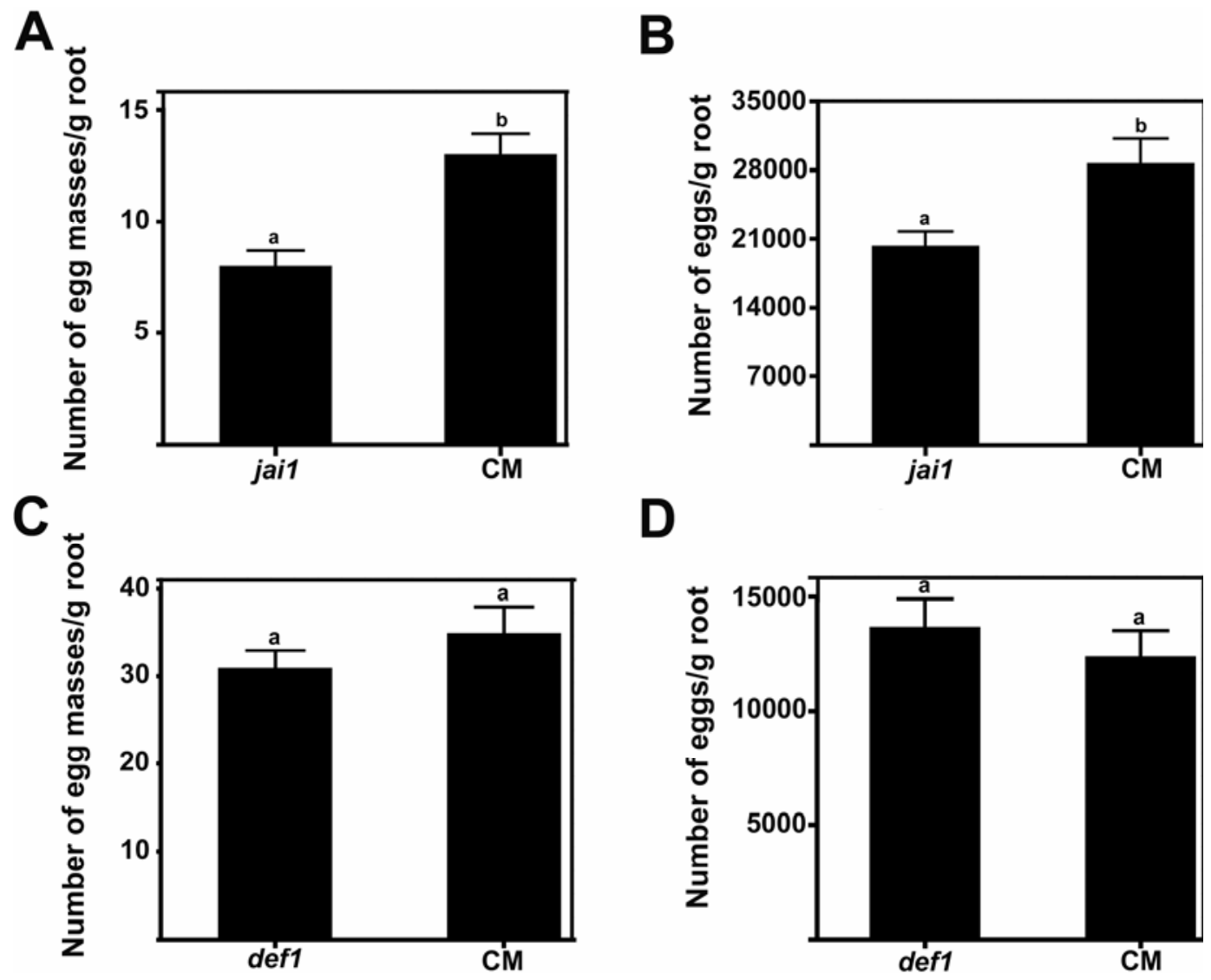

Fig. 5. Nematode reproduction on jail, defl mutants, and wild-type Castlemart. Five-week-old plants were inoculated with 3,000 root-knot nematode secondstage juveniles and plants were evaluated 8 weeks after inoculation. Error bars indicate standard error of the mean, where bars with different letters denote a significant difference at $P<0.001$. A and $\mathbf{C}$, Egg masses per gram of fresh root weight. $\mathbf{B}$ and $\mathbf{D}$, Eggs per gram of fresh root weight. Experiments were performed twice with similar results. Results from one experiment are shown. 
To confirm the role of JA in basal defense to RKNs, jail and $\mathrm{CM}$ plants were further evaluated for RKN parasitism and fecundity. In this experiment, plants were evaluated for both number of egg masses and number of eggs per gram of root. There was a significantly $(P<0.05)$ lower number of nematode egg masses per gram of root on jail than on CM (Fig. 5A). The number of eggs per gram of root was also significantly lower on jail than on CM (Fig. 5B), confirming our earlier results.

To further assess the role of JA in basal resistance to RKNs, we evaluated the JA biosynthetic mutant defl and its wild-type parent $\mathrm{CM}$ for nematode parasitism and fecundity. The defl mutant does not accumulate JA after wounding but the precise nature of the defl mutation is not yet identified. No significant $(P<0.05)$ difference in number of nematode egg masses (Fig. $5 C$ ) and number of eggs (Fig. 5D) per gram of root was observed between defl and the wild-type CM.

SA signaling has been shown to be important for biotic interactions and, because SA and JA signaling could participate in negative cross talk, we questioned whether the reduced susceptibility in the jail mutant is due to increased accumulation of SA (Bostock 2005; Rojo et al. 2003). To examine whether SA accumulated to higher levels in jail plants compared with CM, we performed time-course nematode infections of jail and $\mathrm{CM}$ plants. SA levels in RKN-infected jail and CM roots were not significantly $(P<0.05)$ different during the first 7 days after RKN infections (Fig. 6).

NahG expression did not alter RKN resistance in tomato.

The limited numbers of SA-responsive genes present on the TOM1 chip were differentially regulated in both compatible and incompatible interactions. Previously, RKN growth was observed in an in vitro system with $A$. rhizogenes transgenic hairy roots expressing $N a h G$ in the presence of $M i$ - 1 , indicating a role for SA in $M i-1$ resistance to RKN (Branch et al. 2004). To test the effect of $N a h G$ on $M i-1$ resistance in stably transformed tomato plants in a pot system, we evaluated $\mathrm{Mi}-\mathrm{I}$ $N a h G$ plants and parental types for RKN infection. To assess the role of SA in basal defense to RKNs, NahG and wild-type susceptible parent Moneymaker were also evaluated for RKN infection. Both the number of egg masses (Fig. 7A) and the number of eggs (data not shown) per gram of root on $\mathrm{Mi}-\mathrm{I}$ $N a h G$ plants were not significantly $(P<0.001)$ different than on $M i-1$ plants but were significantly different than on $N a h G$ and Moneymaker. In addition, there were no significant $(P<$ 0.05) differences in the number of egg masses (Fig. 7A) and

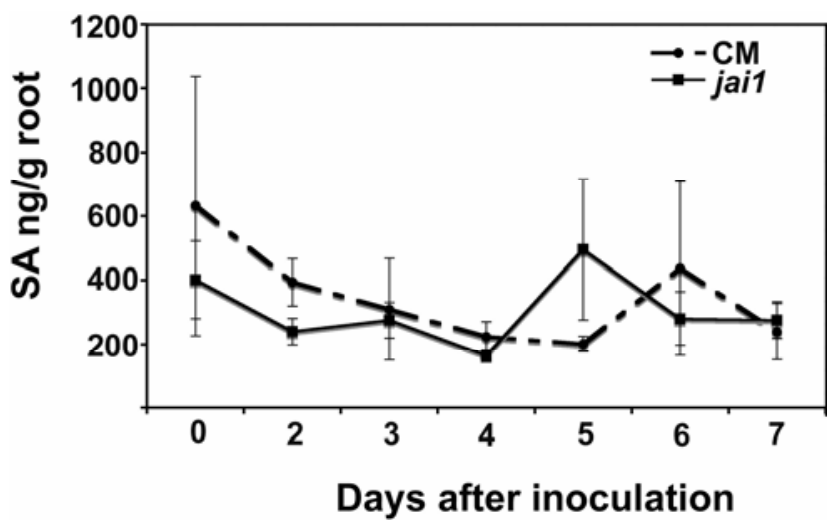

Fig. 6. Similar salicylic acid (SA) levels were detected in jail and wild-type Castlemart (CM) roots. Four-week-old plants were inoculated with 3,000 root-knot nematode second-stage juveniles. Roots were sampled at $0,2,3$, 4, 5, 6, and 7 days after inoculation. Both free SA and bound SA (salicylic acid glucoside) were measured and total SA levels were determined. Three plants per genotype per time point were used. Error bars indicate standard error of the mean. the number of eggs (data not shown) per gram of root on $N a h G$ and Moneymaker plants. To confirm the integrity of $N a h G$ and its ability to convert SA into catechol, a subset of the plants used in the RKN bioassay was used for SA measurements 4 weeks after nematode infection. Significantly $(P<$ $0.001)$ higher levels of total SA were detected in roots of VFN and Moneymaker compared with roots of $M i-1 \quad N a h G$ and $N a h G$ plants (Fig. 7B).

\section{DISCUSSION}

Previous work describing transcriptome analyses of plants infected with RKN primarily focused on understanding the compatible interactions at several days post infection (Bar-Or
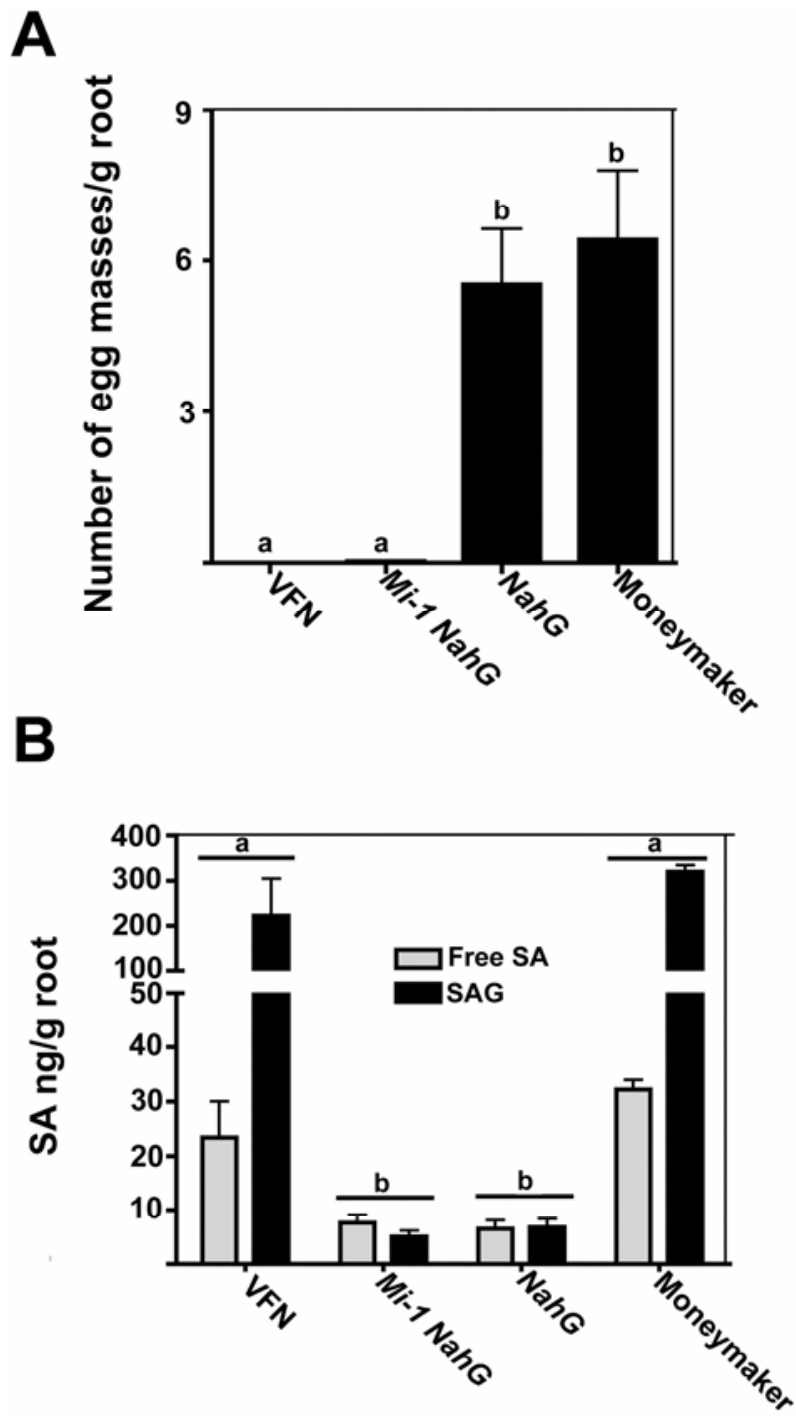

Fig. 7. Nematode reproduction and salicylic acid (SA) content on Mi-1 $N a h G$ plants and wild-type parents. Five-week-old plants were inoculated with 3,000 root-knot nematode (RKN) second-stage juveniles (J2) and plants were evaluated by counting nematode egg masses and eggs on each root. Error bars indicate standard error of the mean (SEM), where bars with different letters denote a significant difference at $P<0.001$. A, Egg masses per gram of fresh root weight. B, Quantification of SA in tomato roots shows that $N a h G$ expression reduces SA accumulation significantly. Five-week-old VFN, Mi-1 NahG, NahG, and Moneymaker plants were inoculated with 3,000 RKN J2. Four weeks after nematode infection, roots were used for SA measurement. Both free SA and bound SA (salicylic acid glucoside $[\mathrm{SAG}]$ ) were measured. Three plants per genotype were used. Error bars indicate SEM. Bars with different letters denote a significant difference at $P<0.001$. 
et al. 2005; Jammes et al. 2005), or used preselected clones (Schaff et al. 2007). In this work, we studied an early time point ( $24 \mathrm{~h}$ after RKN infection) in tomato-RKN compatible and incompatible interactions and, therefore, were able to examine major differences and similarities in transcript profiles in both interactions. Furthermore, the cDNA clones on the microarray used in our study were not selected for plant nematode interactions and, therefore, represent an unbiased selection of genes.

Our microarray analyses using tomato root tissue highly enriched for RKN infection indicated that roots undergo substantial reprogramming in both compatible and incompatible tomato-RKN interactions. Similarly, the upregulation of ribosomal genes in both interactions indicated a major role for protein synthesis in RKN-infected roots. The large number of common genes regulated in both compatible and incompatible interactions also indicated considerable overlap in plant responses. A large portion of these genes, over $34 \%$, is of unknown function and may assist in identifying novel components of plant defense.

The hallmark of RKN feeding is the establishment of the feeding site, or the giant cells, which are characterized by unusual hypermitotic activities. Our data indicated that, in both compatible and incompatible interactions, cyclin and cyclindependent protein kinase were upregulated by the nematode, suggesting that RKN triggers the initiation of the feeding site in resistant tomato. In addition to accelerated cell division, giant cells demonstrate aneuploidy (Wiggers et al. 1990). In the incompatible response, the differential regulation of Dis3 (defective in sister chromatid disjoining 3), a gene required for proper kinetochore formation and chromosome segregation (Murakami et al. 2007), suggested that a proper control of chromosome segregation could be a part of plant defense against RKNs.

One of the earliest detectable cellular events in plant cells challenged with pathogens is the ion fluxes through the plasma membrane. These include $\mathrm{Ca}^{+}$ions that have a role in regulating a myriad of cellular responses in eukaryotes, including plant defense responses mediated by $R$ genes. Calmodulin is a highly conserved and well-characterized $\mathrm{Ca}^{+}$sensor and is involved in $\mathrm{Ca}^{+}$signaling by modulating a variety of enzymes and proteins (Bouche et al. 2005; David et al. 2005). In this study, calmodulin was differentially upregulated during the incompatible interactions, supporting a role for $\mathrm{Ca}^{+}$signaling in $M i-1$ defense to RKN.

Together with ion influx and oxidative burst, $R$-gene-mediated resistance is frequently associated with HR, as is the case for the Mi-1 resistance to RKN. Necrotic cells are commonly seen in the incompatible interaction within $24 \mathrm{~h}$ of nematode infection. Thus, in the presence of necrosis, it is expected that Hsr201 and Hsr203, reported to be induced by HR, would be upregulated (Pierre et al. 1996; Takahashi et al. 2004). The differential upregulation of these genes in the incompatible interaction indicated that our nematode inoculation system is appropriate for this study and further confirmed that $M i-1$ resistance to RKN involved early induction of HR.

Another noteworthy group of differentially regulated genes were involved in transcription (9\%) (Fig. 3). Among these were transcription factors involved in plant development (e.g., MADS box, homeobox, and NAC) (Bolle 2004; Gong et al. 2004) and in a number of biotic and abiotic stress signal transduction (e.g., MYB, AP2, WRKY, and bZIP) (Chen et al. 2002; Eulgem 2005). Among the WRKY transcription factors, WRKY6, WRKY61, and a WRKY72-like gene were specifically upregulated during incompatible interaction whereas WRKY3, WRKY23, and a WRKY33-like gene were expressed during both compatible and incompatible interactions, indicat- ing differential roles for these structurally different families of transcription factors in RKN defense.

Earlier studies have shown that nematode infection activates $P R$ genes during compatible interactions (Bar-Or et al. 2005; Gheysen and Fenoll 2002; Lambert et al. 1999). In agreement with these findings, our study indicated that a number of $P R$ genes were induced in both compatible and incompatible interactions, suggesting considerable overlap between these two interactions (Fig. 3). Among the defense-related genes upregulated in both interactions are Pin1 and Pin2. Therefore, the upregulation of these genes by nematode infection may implicate the octadecanoid signaling pathway in nematode resistance. Despite the activation of these wound-induced genes, no role for JA signaling was identified in $M i-1$ resistance to RKNs. The high number of nematodes used in our system may cause wounding leading to activation of Pin1 and Pin2. We are unable to confirm this possibility because previous gene-expression studies performed with tomato and RKNs either did not use early-infection time points or did not have these genes or other wound-induced genes on their arrays. Earlier, we also reported the upregulation of both Pin1 and Pin2 by aphid feeding in both compatible and incompatible interactions (Bhattarai et al. 2007b; Martinez de Ilarduya et al. 2003), suggesting common tomato defense responses to aphids and RKNs. However, as is the case for RKN, introducing the jail mutation in the $M i-1$ background did not attenuate the $M i-1$ resistance to potato aphids, indicating no role for JA signaling in the $\mathrm{Mi}-1$ resistance to aphids (Bhattarai et al. 2007b).

Previously, we investigated the role of JA in the tomato basal resistance to aphids using the jail mutant (Bhattarai et al. 2007b). Aphid survival and fecundity were similar on the jail mutant and wild-type parent, indicating no role for JA in aphid defense in tomato. In contrast, the jail mutant showed reduced susceptibility to RKNs compared with the wild-type parent. However, reduced susceptibility was not observed with the JA biosynthetic mutant defl, indicating that nematode susceptibility does not depend on JA biosynthesis but requires an intact Coi- 1 signaling pathway. This observation is similar to a previously reported tomato-Pseudomonas syringae pv. tomato interaction, where virulence to $P$. syringae required an intact Coi-1 signaling pathway (Zhao et al. 2003). Therefore, it is intriguing to speculate that, similar to $P$. syringae, RKNs may produce a JA functional analog like the phytotoxin coronatine and may target the Coi-1 signaling pathway (Bender et al. 1999; Weiler et al. 1994). The genomes of two RKN species, Meloidogyne incognita and M. hapla, are currently being sequenced and these projects may assist in identifying the existence of JA analogs in nematodes.

The decrease in RKN reproduction on jail plants and activation of JA-responsive genes by RKN feeding might indicate that the nematode is able to manipulate plant defense responses to its advantage by leveraging the existing cross talk between JA and SA signaling pathways (Bostock 2005; Rojo et al. 2003). However, the lack of increase in SA levels in jail roots compared with the wild-type parent, both before and after RKN infection, could not confirm this antagonism of plant defenses by RKN. The lack of increases in SA levels in the jail mutant could be because the cross talk between SA and JA pathways is downstream of SA. Alternatively, the inability to detect an increase in the SA levels in RKN-infected jail roots could be due to the lack of synchronized RKN infection in the assay system used. Unlike infections with microbial and viral pathogens where it is relatively easy to get synchronized infections that trigger abrupt plant responses, nematode infections are much slower, making it difficult to determine exactly when plant responses occur. It is also possible that slight increases in SA levels could be sufficient to heighten plant defenses to RKNs. 
In agreement with the later possibility is our result with the transgenic tomato $N a h G$ line. Compared with the wild-type parent Moneymaker, $N a h G$ tomato did not show increased susceptibility to RKN infection, suggesting that the residual level of SA present in this tomato line is sufficient for basal resistance to RKNs. Similarly, low levels of SA might be sufficient for $\mathrm{Mi}-1$ resistance to RKNs because $\mathrm{Mi}-1 \mathrm{NahG}$ plants were completely resistant to RKNs. A previous report using transgenic hairy roots containing $\mathrm{Mi}-\mathrm{l}$ and expressing $\mathrm{NahG}$ showed some RKN development on these roots (Branch et al. 2004). The number of nematodes developing on these roots was lower than those on the near-isogenic tomato Moneymaker, indicating partial loss of resistance. The discrepancy in our results could be due to the different systems used (transgenic hairy roots in tissue culture versus transgenic plant and pot system). Alternatively, the system used by Branch and associates (2004) degraded SA more efficiently and reduced the pool of free SA in the roots. Indeed, free SA levels in the transgenic hairy roots with $M i-1$ were lower than what we report here (V. M. Williamson, personal communication). The lower levels of free SA in the hairy root system could be due to an enhanced $35 \mathrm{~S}$ promoter used, or cultured hairy roots with higher expression of $N a h G$ assisting in quicker degradation of SA. Regardless, these results suggested that low levels of SA were sufficient for both basal and $M i-1$ resistance to RKNs. A role for SA in basal and $M i-1$ resistance to potato aphids was reported earlier, further supporting the idea that $M i-1$ resistances required SA and demonstrating common plant defenses against RKNs and potato aphids (Li et al. 2006).

Auxin has been implicated in giant cell and gall formation by RKNs, perhaps by regulating the expression and activity of cell cycle genes in the giant cells or galls (Bird and Koltai 2000; Goverse et al. 2000). Our microarray analysis indicated that auxin-related genes were also differentially expressed in both compatible and incompatible interactions. An earlier report indicated that RKNs failed to penetrate and establish a feeding site on an auxin-insensitive tomato mutant (Richardson and Price 1984), suggesting a direct role for auxin signaling in susceptibility of tomato to RKN. This may be due to inhibition of giant cell formation by affecting the cell cycle (Bird and Koltai 2000; Goverse et al. 2000), increased plant defense (Navarro et al. 2006), or both. The JA-dependent signaling also interacts with an auxin-dependent signaling pathway (Saniewski et al. 2002). Although the jail mutant does not display an obvious auxin mutant phenotype, it is unclear whether this mutant has altered sensitivity to auxin and whether this alteration in auxin sensitivity can lead to reduction in RKN infection. Future experiments will explore this possibility.

\section{MATERIALS AND METHODS}

\section{Plant materials and growth conditions.}

A pair of nearly isogenic RKN-resistant and -susceptible tomato cultivars, Motelle $(\mathrm{Mi}-\mathrm{l} / \mathrm{Mi}-\mathrm{l})$ and Moneymaker $(\mathrm{mi} / \mathrm{mi})$, respectively, were used for the microarray experiments. VFN (Mi-1/Mi-1), CM ( $\mathrm{mi} / \mathrm{mi})$, a jail mutant in CM background (Li et al. 2004), a cross between VFN and jail (Mi-1 jail) (Bhattarai et al. 2007b), a defl mutant in CM background, $N a h G$ in Moneymaker background $(N a h G)$, and a cross between VFN and $N a h G(M i-1 ~ N a h G)$ (Li et al. 2006) were also used.

Unless otherwise stated, seed were treated with $10 \%$ (vol/vol) bleach and germinated in organic planting mix (SunGro Horticulture, Bellevue, WA, U.S.A.) in seedling trays. Seedlings were maintained in growth chambers at $24^{\circ} \mathrm{C}$ with a photoperiod of 16 and $8 \mathrm{~h}$ at $200 \mu \mathrm{mol} \mathrm{m} \mathrm{m}^{-2} \mathrm{~s}^{-1}$. Two weeks after germination, seedlings were transplanted into pots $(10 \mathrm{~cm}$ in diameter and $17 \mathrm{~cm}$ deep) filled with UC mix and sand (1:4, vol/vol) supplemented with Osmocote (Sierra Chemical Company, Milpitas, CA, U.S.A.). Plants were maintained in a greenhouse at 24 to $26^{\circ} \mathrm{C}$ and fertilized bimonthly with MiracleGro (Stern's MiracleGro Products, Port Washington, NY, U.S.A.). Plants with $N a h G$ were maintained in a growth chamber at $25^{\circ} \mathrm{C}$ with a photoperiod of 16 and $8 \mathrm{~h}$ at $60 \mu \mathrm{mol} \mathrm{m} \mathrm{m}^{-2} \mathrm{~s}^{-1}$. Plants grown under this condition didn't develop necrotic lesions.

\section{Selection of mutants and crosses.}

A heterozygous population of the jail mutant and F2 population of VFN $\times$ jail were screened for methyl jasmonate sensitivity and genotyped for the presence of the jail deletion as described by Bhattarai and associates (2007b). In Mi-1 NahG plants, the presence of $N a h G$ transgene was confirmed by PCR. In F2 populations of VFN $\times$ jail and $\mathrm{VFN} \times N a h G$, the presence of $M i-1$ was identified using the codominant marker REX-1 as described by Williamson and associates (1994), and plants homozygous for $M i-1$ were selected.

\section{Nematode isolate and inoculum preparation.}

$M$. javanica isolate VW4 and $M$. incognita isolate P77R3, maintained on susceptible tomato $\mathrm{cv}$. UC82B, were used. Nematode eggs were collected by bleach extracting the roots following established protocols (Hussey and Barker 1973). Eggs were allowed to hatch using modified Baermann funnels (Martinez de Ilarduya et al. 2001). After 2 days, J2 were collected and used in bioassays. For microarray samples, the J2 were concentrated using a filtration apparatus fitted with a 1$\mu \mathrm{m}$ polycarbonate membrane (GE Osmonics Labstore, Minnetonka, MN, U.S.A.) and washed with sterile water (Lambert et al. 1999). To further clean the nematodes, the J2 were transferred onto a sand column lined with tissue papers. After $24 \mathrm{~h}$, $\mathrm{J} 2$ that migrated through the column were collected, concentrated as described above, and suspended in sterile water.

\section{Root infections for microarray.}

Tomato seed were surface sterilized in $10 \%$ (vol/vol) bleach and germinated in sterile conditions in the dark. Seedlings were grown on Whatman paper in plastic containers and nematode infections were carried out as described by Lambert and associates (1999). Seedlings with approximately $1.5-\mathrm{cm}$ root length were infected with 100 to $150 \mathrm{~J} 2$ in sterile $0.5 \%$ (wt/vol) carboxymethyl cellulose (Sigma-Aldrich, St. Louis). Control seedlings were inoculated with the same volume of $0.5 \%(\mathrm{wt} / \mathrm{vol})$ carboxymethyl cellulose. Approximately $24 \mathrm{~h}$ after inoculation, $1 \mathrm{~cm}$ of the infected root tips was excised, quickly frozen, and stored at $-80^{\circ} \mathrm{C}$. A few seedlings were stained in acid fuchsin to check the nematode infection rate. The described inoculations were performed four times. All components used in this system were autoclaved before use and all manipulations were performed aseptically in a laminar flow hood.

\section{RNA isolation.}

RNA was isolated using hot phenol. Frozen roots were ground in liquid nitrogen and extracted by vigorous vortexing for $30 \mathrm{~s}$ in a mixture of water-saturated phenol and an equal volume of extraction buffer (100 mM Tris- $\mathrm{HCl}, \mathrm{pH} 8.0 ; 100$ $\mathrm{mM} \mathrm{LiCl} ; 10 \mathrm{mM}$ EDTA; and 1\% wt/vol sodium dodecyl sulfate [SDS]) preheated at $80^{\circ} \mathrm{C}$. Sample was then extracted with chloroform and RNA was precipitated by adding an equal volume of $4 \mathrm{M} \mathrm{LiCl}$ and incubating at $-70^{\circ} \mathrm{C}$ for $30 \mathrm{~min}$. RNA was pelleted by centrifugation at $12,000 \times g$ for $20 \mathrm{~min}$ at $4^{\circ} \mathrm{C}$, washed with $70 \%$ ( $\mathrm{vol} / \mathrm{vol}$ ) ethanol, and resuspended in nuclease-free water, then treated with DNase I (Promega Corp., Madison, WI, U.S.A.) and purified with RNeasy mini kit (Qiagen, Valencia, CA, U.S.A.). 


\section{Microarray fabrication.}

The tomato cDNA microarray chip (TOM1) was used for this experiment. This chip contains 12,860 EST clones which represent 8,642 independent tomato loci (Bonshtien et al. 2005). Tomato TOM1 microarray fabrication has been described by Alba and associates (2004) and the EST libraries utilized for the arrays have been described by Van der Hoeven and associates (2002).

\section{Target labeling.}

DNase I-treated and RNeasy-purified total RNAs $(20 \mu \mathrm{g}$ each) were used to generate cDNA in $30 \mu \mathrm{l}$ with $6 \mu \mathrm{g}$ of random hexamers, $5 \times$ first-strand buffer, $10 \mathrm{mM}$ dithiothreitol, and 400 units of Superscript II Reverse Transcriptase, 50x aminoallyl-dNTP mix (Invitrogen, Carlsbad, CA, U.S.A.). Unincorporated amino acid-dUTP and free amines were removed and cDNA were labeled with monofunctional NHSester $\mathrm{Cy} 3$ or $\mathrm{Cy} 5$ flurophores (Amersham, Piscataway, NJ, U.S.A.). Infected samples were labeled with Cy5 and uninfected samples were labeled with $\mathrm{Cy} 3$. Uncoupled dye was removed using the Qiagen PCR Purification kit (Qiagen). Labeling quality and quantity were assessed using a spectrophotometer.

\section{Hybridization, washes, and scanning.}

Slides were prehybridized in a buffer containing $5 \times \operatorname{SSC}(1 \times$ $\mathrm{SSC}$ is $0.15 \mathrm{M} \mathrm{NaCl}$ plus $0.015 \mathrm{M}$ sodium citrate), $0.1 \%$ (wt/vol) SDS, $1 \%$ (wt/vol) bovine serum albumen, and $10 \mathrm{mM}$ EDTA for $45 \mathrm{~min}$ at $42^{\circ} \mathrm{C}$, followed by two washes in $0.1 \times$ $\mathrm{SSC}$ at room temperature. Equal amounts of $\mathrm{Cy} 3$ and $\mathrm{Cy} 5-$ labeled probes, for a total volume of $17 \mu \mathrm{l}, 2 \mu \mathrm{l}$ of $0.5 \mathrm{M}$ EDTA, and $1 \mu \mathrm{l}$ of Poly(A)-DNA $(20 \mu \mathrm{g} / \mu \mathrm{l})$ (Amersham) were mixed with an equal volume of the hybridization buffer for a total of $40 \mu \mathrm{l}$. The hybridization buffer contained $50 \%$ (vol/ vol) formamide, $5 \times \mathrm{SSC}$, and $0.1 \%$ (wt/vol) SDS. Hybridization was carried out in hybridization chambers (Corning, Corning, NY, U.S.A.) at $42^{\circ} \mathrm{C}$ for $16 \mathrm{~h}$ in the dark. Posthybridization washes were carried out at room temperature according to the manufacturer's instructions and slides were dried. ScanArray Express (PerkinElmer Life and Analytical Sciences, Waltham, MA, U.S.A.) was used to scan the slides to collect the images. Separate images for each fluorescence were acquired. Image files were quantified using Imagene 5.6 software (Biodiscovery, El Segundo, CA, U.S.A.). In default setting, individual intensity files were created for $\mathrm{Cy} 3$ and $\mathrm{Cy} 5$ for both infected and uninfected samples of both tomato genotypes.

\section{Data analysis.}

The raw intensity data were imported into the $\mathrm{R}$ software and analyzed with BioConductor packages (Gentleman et al. 2005; R Project website). The intensity values were normalized with the print-tip LOWESS approach and differentially expressed genes were identified with the Limma package (Smyth 2005). Genes were classified as differentially expressed on the basis of an adjusted $P$ value of $<0.05$. False discovery rate $(5 \%)$ was used to correct the raw $P$ values for multiple testing. Functional classification of the differentially expressed genes was based on Solanaceae Genomics Network SGN and Gene Ontology Consortium annotations.

\section{Reverse-transcription real-time qPCR.}

DNase I-treated 2.5- $\mu \mathrm{g}$ total RNAs were reverse transcribed using oligo dT primers and the Thermoscript RT-PCR system (Invitrogen) in a $20-\mu l$ volume following the manufacturer's recommendation. Gene-specific primers were designed using Primer3 software (Rozen and Skaletsky 2000). The iQ SYBR
Green Supermix (Biorad, Hercules, CA, U.S.A.) was used as intercalating dye to detect the amplification product in iCyclcler5 IQ (Biorad). PCR was carried out in $20 \mu \mathrm{l}$ using 2 $\mu \mathrm{l}$ of $10 \times$ diluted cDNA as template. Three technical replicates and two biological replicates were performed for each gene and the expression level of genes were determined relative to that of Ubi3 (Ginzinger 2002).

\section{Nematode bioassays.}

Five-week-old plants were individually infected with 3,000 J2 (Martinez de Ilarduya et al. 2001). Plants were maintained at 22 to $26^{\circ} \mathrm{C}$ in a greenhouse for 8 weeks. Later, roots were stained in $0.001 \%$ (wt/vol) erioglaucine (Sigma-Aldrich) and nematode egg masses were counted. Nematode eggs were extracted from individual roots as described earlier (Hussey and Barker 1973). The number of eggs per sample was counted in three 1-ml aliquots. For each experiment, 20 plants per genotype were used. The experiment was performed twice.

\section{Nematode time-course infections.}

Four-week-old jail and CM seedlings, grown in pots $(5 \mathrm{~cm}$ in diameter and $6 \mathrm{~cm}$ deep) and maintained in a growth chamber, were infected with 3,000 J2. Roots were sampled at 0, 2, $3,4,5,6$, and 7 days after infection and frozen immediately in liquid nitrogen. Three roots per time-point or genotype were collected and used for SA quantification.

\section{SA quantification.}

Root samples were ground to a fine powder with a mortar and pestle in liquid nitrogen. For each sample, SA concentration was measured in duplicate as described by $\mathrm{Li}$ and associates (2006). Both free SA and salicylic acid glucoside (SAG) were measured. SAG was determined by quantifying the free SA released after acid hydrolysis of the bound fraction.

\section{Statistical analysis.}

For statistical analysis of nematode bioassays, the statistical package SAS (v. 9.1 for Windows; SAS Institute Inc., Cary, NC, U.S.A.) was used. Data having three or more treatments were analyzed using analysis of variance. Pairwise comparisons were performed using Fisher's probable least-squares differences. For the time course SA level measurement, paired comparisons within individual days were performed using a $t$ test.

\section{ACKNOWLEDGMENTS}

We thank the L. Walling Lab members (University of California [UC], Riverside) and V. M. Williamson (UC Davis) for helpful discussions. We also thank T. Eulgem (UC Riverside) for discussions and helpful comments on the manuscript, H. Jin (UC Riverside) for advice on qPCR, and J. Borneman (UC Riverside) for access to the Icycler in his laboratory. This work was supported by grants from University of California Agricultural Experiment Station and the National Science Foundation (IOB0543937) to I. Kaloshian.

\section{LITERATURE CITED}

Alba, R., Fei, Z., Payton, P., Liu, Y., Moore, S. L., Debbie, P., Cohn, J., D’Ascenzo, M., Gordon, J. S., Rose, J. K. C., Martin, G., Tanksley, S. D., Bouzayen, M., Jahn, M. M., and Giovannoni, J. J. 2004. ESTs, cDNA microarrays, and gene expression profiling: Tools for dissecting plant physiology and development. Plant J. 39:697-714.

Bar-Or, C., Kapulnik, Y., and Koltai, H. 2005. A broad characterization of the transcriptional profile of the compatible tomato response to the plant parasitic root knot nematode Meloidogyne javanica. Eur. J. Plant Pathol. 111:181-192.

Bender, C. L., Alarcon-Chaidez, F., and Gross, D.C. 1999. Pseudomonas syringae phytotoxins: Mode of action, regulation, and biosynthesis by peptide and polyketide synthetases. Microbiol. Mol. Biol. Rev. 63:266-292. 
Bhattarai, K. K., Li, Q., Liu, Y., Dinesh-Kumar, S. P., and Kaloshian, I. 2007a. The Mi-1-mediated pest resistance requires Hsp90 and Sgt1. Plant Physiol. 144:312-323.

Bhattarai, K. K., Xie, Q.-G., Pourshalimi, D., Younglove, T., and Kaloshian, I. 2007b. Coil-dependent signaling pathway is not required for $\mathrm{Mi}$-1mediated potato aphid resistance. Mol. Plant-Microbe Interact. 20:276282

Bird, D. M., and Koltai, H. 2000. Plant parasitic nematodes: Habitats, hormones, and horizontally-acquired genes. J. Plant Growth Regul. 19:183-194.

Bolle, C. 2004. The role of GRAS proteins in plant signal transduction and development. Planta 218:683-692.

Bonshtien, A., Lev, A., Gibly, A., Debbie, P., Avni, A., and Sessa, G. 2005. Molecular properties of the Xanthomonas AvrRxv effector and global transcriptional changes determined by its expression in resistant tomato plants. Mol. Plant-Microbe Interact. 18:300-310.

Bostock, R. 2005. Signal crosstalk and induced resistance: Straddling the line between cost and benefit. Annu. Rev. Phytopathol. 43:545-580.

Bouche, N., Yellin, A., Snedden, W. A., and Fromm, H. 2005. Plant-specific calmodulin-binding proteins. Annu. Rev. Plant Biol. 56:435-466.

Branch, C., Hwang, C. F., Navarre, D. A., and Williamson, V. M. 2004. Salicylic acid is part of the $\mathrm{Mi}-1$-mediated defense response to rootknot nematode in tomato. Mol. Plant-Microbe Interact. 17:351-356.

Chen, W., Provart, N. J., Glazebrook, J., Katagiri, F., Chang, H. S., Eulgem, T., Mauch, F., Luan, S., Zou, G., Whitham, S. A., Budworth, P. R., Tao, Y., Xie, Z., Chen, X., Lam, S., Kreps, J.A., Harper, J. F., SiAmmour, A., Mauch-Mani, B., Heinlein, M., Kobayashi, K., Hohn, T., Dangl, J. L., Wang, X., and Zhu, T. 2002. Expression profile matrix of Arabidopsis transcription factor genes suggests their putative functions in response to environmental stresses. Plant Cell 14:559-574.

David, C., Sophia, K. E., Gregory, B. M., Stephanie, L. D., and Wayne, A. S. 2005. Calmodulin-like proteins from Arabidopsis and tomato are involved in host defense against Pseudomonas syringae pv. tomato. Plant Mol. Biol. 58:887-897.

Dropkin, V. H. 1969. Cellular responses of plants to nematode infections. Annu. Rev. Phytopathol. 7:101-122.

Eulgem, T. 2005. Regulation of the Arabidopsis defense transcriptome. Trends Plant Sci. 10:71-78.

Gentleman, R., Carey, V., Dudoit, S., Irizarry, R., and Huber, W. 2005. Bioinformatics and Computational Biology Solutions Using $\mathrm{R}$ and Bioconductor. Springer, New York.

Gheysen, G., and Fenoll, C. 2002. Gene expression in nematode feeding sites. Annu. Rev. Phytopathol. 40:191-219.

Ginzinger, D. G. 2002. Gene quantification using real-time quantitative PCR An emerging technology hits the mainstream. Exp. Hematol. 30:503-512.

Glazebrook, J. 2005. Contrasting mechanisms of defense against biotrophic and necrotrophic pathogens. Annu. Rev. Phytopathol. 43:205-227.

Gong, W., Shen, Y. P., Ma, L. G., Pan, Y., Du, Y. L., Wang, D. H., Yang, J. Y., Hu, L. D., Liu, X. F., Dong, C. X., Ma, L., Chen, Y. H., Yang, X. Y., Gao, Y., Zhu, D., Tan, X., Mu, J. Y., Zhang, D. B., Liu, Y. L., DineshKumar, S. P., Li, Y., Wang, X. P., Gu, H. Y., Qu, L. J., Bai, S. N., Lu, Y. T., Li, J. Y., Zhao, J. D., Zuo, J., Huang, H., Deng, X. W., and Zhu, Y. X. 2004. Genome-wide ORFeome cloning and analysis of Arabidopsis transcription factor genes. Plant Physiol. 135:773-782.

Goverse, A., de Engler, J. A., Verhees, J., van der Krol, S., Helder, J. H., and Gheysen, G. 2000. Cell cycle activation by plant parasitic nematodes. Plant Mol. Biol. 43:747-761.

Hammond-Kosack, K. E., and Parker, J. E. 2003. Deciphering plant-pathogen communication: Fresh perspectives for molecular resistance breeding. Curr. Opin. Biotechnol. 14:177-193.

Hussey, R., and Barker, K. R. 1973. A comparison of methods of collecting inocula of Meloidogyne species including a new technique. Plant Dis. Rep. 57:1025-1028.

Jammes, F., Lecomte, P., de Almeida-Engler, J., Bitton, F., MartinMagniette, M. L., Renou, J. P., Abad, P., and Favery, B. 2005. Genomewide expression profiling of the host response to root-knot nematode infection in Arabidopsis. Plant J. 44:447-458.

Koenning, S. R., Overstreet, C., Noling, J. W., Donald, P. A., Becker, J. O., and Fortnum, B. A. 1999. Survey of crop losses in response to phytoparasitic nematodes in the United States for 1994. J. Nematol. 13:587-618.

Kunkel, B. N., and Brooks, D. M. 2002. Cross talk between signaling pathways in pathogen defense. Curr. Opin. Plant Biol. 5:325-331.

Lambert, K. N., Ferrie, B. J., Nombela, G., Brenner, E. D., and Williamson, V. M. 1999. Identification of genes whose transcripts accumulate rapidly in tomato after root-knot nematode infection. Physiol. Mol. Plant Pathol. 55:341-348

Li, L., Li, C. Y., and Howe, G. A. 2001. Genetic analysis of wound signaling in tomato. Evidence for a dual role of jasmonic acid in defense and female fertility. Plant Physiol. 127:1414-1417.

Li, L., Zhao, Y., McCaig, B. C., Wingerd, B. A., Wang, J., Whalon, M. E.,
Pichersky, E., and Howe, G. A. 2004. The tomato homolog of CORONATINE-INSENSITIVE1 is required for the maternal control of seed maturation, jasmonate-signaled defense responses, and glandular trichome development. Plant Cell 16:126-143.

Li, Q., Xie, Q.-G., Smith-Becker, J., Navarre, D. A., and Kaloshian, I. 2006. Mi-1-mediated aphid resistance involves salicylic acid and mitogen-activated protein kinase signaling pathways. Mol. Plant-Microbe Interact. 19:655-664.

Martinez de Ilarduya, O., Moore, A. E., and Kaloshian, I. 2001. The tomato Rme 1 locus is required for $M i-1$-mediated resistance to root-knot nematodes and the potato aphid. Plant J. 27:417-425.

Martinez de Ilarduya, O., Xie, Q.-G., and Kaloshian, I. 2003. Aphidinduced defense responses in $\mathrm{Mi}$-1-mediated compatible and incompatible tomato interactions. Mol. Plant-Microbe Interact. 16:699-708.

Martinez de Ilarduya, O., Nombela, G., Hwang, C. F., Williamson, V. M., Muñiz, M., and Kaloshian, I. 2004. Rme1 is necessary for Mi-1-mediated resistance and acts early in the resistance pathway. Mol. PlantMicrobe Interact. 17:55-61.

Milligan, S. B., Bodeau, J., Yaghoobi, J., Kaloshian, I., Zabel, P., and Williamson, V. M. 1998. The root-knot nematode resistance gene $M i$ from tomato is a member of leucine zipper, nucleotide binding, leucinerich repeat family of plant genes. Plant Cell 10:1307-1319.

Murakami, H., Goto, D. B., Toda, T., Chen, E. S., Grewal, S. I. Martienssen, R. A., and Yanagida, M. 2007. Ribonuclease activity of Dis 3 is required for mitotic progression and provides a possible link between heterochromatin and kinetochore function. PLoS ONE 2:e317.

Navarro, L., Zipfel, C., Rowland, O., Keller, I., Robatzek, S., Boller, T., and Jones, J. D. 2004. The transcriptional innate immune response to flg22. Interplay and overlap with $A v r$ gene-dependent defense responses and bacterial pathogenesis. Plant Physiol. 135:1113-1128.

Navarro, L., Dunoyer, P., Jay, F., Arnold, B., Dharmasiri, N., Estelle, M., Voinnet, O., and Jones, J. D. 2006. A plant miRNA contributes to antibacterial resistance by repressing auxin signaling. Science 312:436439.

Nombela, G., Williamson, V. M., and Muñiz, M. 2003. The root-knot nematode resistance gene $\mathrm{Mi}-1.2$ of tomato is responsible for resistance against the whitefly Bemisia tabaci. Mol. Plant-Microbe Interact. 16:645-649.

Pierre, C., Hsiou Chen, H., and Yves, M. 1996. Characterization of hsr201 and $h s r 515$, two tobacco genes preferentially expressed during the hypersensitive reaction provoked by phytopathogenic bacteria. Plant Mol. Biol. 31:255-265.

Puthoff, D. P., Nettleton, D., Rodermel, S. R., and Baum, T. J. 2003. Arabidopsis gene expression changes during cyst nematode parasitism revealed by statistical analyses of microarray expression profiles. Plant J. 33:911-921.

Richardson, L., and Price, N. S. 1984. Observations on the biology of Meloidogyne incognita and the diageotropica tomato mutant. Rev. Nematol. 7:97-99.

Rojo, E., Solano, R., and Sanchez-Serrano, J. J. 2003. Interactions between signaling compounds involved in plant defense. J. Plant Growth Regul. 22:82-98.

Rossi, M., Goggin, F. L., Milligan, S. B., Kaloshian, I., Ullman, D. E., and Williamson, V. M. 1998. The nematode resistance gene $M i$ of tomato confers resistance against the potato aphid. Proc. Natl. Acad. Sci. U.S.A. 95:9750-9754

Rozen, S., and Skaletsky, H. J. 2000. Primer3 on the WWW for general users and for biologist programmers. Pages 365-386 in: Bioinformatics Methods and Protocols: Methods in Molecular Biology. S. Krawetz and S. Misener, eds. Humana Press, Totowa, NJ, U.S.A.

Saniewski, M., Ueda, J., and Miyamoto, K. 2002. Relationships between jasmonates and auxin in regulation of some physiological processes in higher plants. Acta Physiol. Plant. 24:211-220.

Schaff, J. E., Nielsen, D. M., Smith, C. P., Scholl, E. H., and Bird, D. M. 2007. Comprehensive transcriptome profiling in tomato reveals a role for glycosyltransferase in $M i$-mediated nematode resistance. Plant Physiol. 144:1079-1092.

Smyth, G. K. 2005. Limma: Linear models for microarray data. Pages 397-420 in: Bioinformatics and Computational Biology Solutions Using R and Bioconductor. R. Gentleman, V. Carey, S. Dudoit, R. Irizarry, and W. Huber, eds. Springer, New York.

Takahashi, Y., Uehara, Y., Berberich, T., Ito, A., Saitoh, H., Miyazaki, A., Terauchi, R., and Kusano, T. 2004. A subset of hypersensitive response marker genes, including HSR203J, is the downstream target of a spermine signal transduction pathway in tobacco. Plant J. 40:586-595.

Van der Hoeven, R., Ronning, C., Giovannoni, J., Martin, G., and Tanksley, S. 2002. Deductions about the number, organization, and evolution of genes in the tomato genome based on analysis of a large expressed sequence tag collection and selective genomic sequencing. Plant Cell 14:1441-1456. 
Weiler, E. W., Kutchan, T. M., Gorba, T., Brodschelm, W., Niesel, U., and Bublitz, F. 1994. The Pseudomonas phytotoxin coronatine mimics octadecanoid signalling molecules of higher plants. FEBS (Fed. Eur. Biochem. Soc.) Lett. 345:9-13.

Wiggers, R. J., Starr, J. L., and Price, H. J. 1990. DNA content and variation in chromosome-number in plant-cells affected by Meloidogyne incognita and M. arenaria. Phytopathology 80:1391-1395.

Williamson, V. M., and Gleason, C. A. 2003. Plant-nematode interactions. Curr. Opin. Plant Biol. 6:327-333.

Williamson, V. M., and Kumar, A. 2006. Nematode resistance in plants: The battle underground. Trends Genet. 22:396-403.

Williamson, V. M., Ho, J.-Y., Wu, F. F., Miller, N., and Kaloshian, I. 1994. A PCR-based marker tightly linked to the nematode resistance gene, $M i$, in tomato. Theor. Appl. Genet. 87:757-763.
Zhao, Y., Thilmony, R., Bender, C. L., Schaller, A., He, S. Y., and Howe, G. A. 2003. Virulence systems of Pseudomonas syringae pv. tomato promote bacterial speck disease in tomato by targeting the jasmonate signaling pathway. Plant J. 36:485-499.

\section{AUTHOR-RECOMMENDED INTERNET RESOURCES}

Boyce Thompson Institute for Plant Research Center of Gene Expression Profiling website: bti.cornell.edu/CGEP/CGEP.html

TIGR Solanaceae Genomics Resource and Microarray Protocols, website: www.tigr.org/tdb/potato/microarray_SOPs.shtml

The R Project for Statistical Computing website: www.R-project.org

Cornell University's Tomato Expression database: ted.bti.cornell.edu 